\title{
Bounded holomorphic functions with multiple sheeted pluripolar hulls
}

\author{
by \\ Armen Edigarian, Józef Siciak and \\ WŁODZIMIERZ ZWONEK (Kraków)
}

\begin{abstract}
We describe compact subsets $K$ of $\partial \mathbb{D}$ and $\mathbb{R}$ admitting holomorphic functions $f$ with the domains of existence equal to $\mathbb{C} \backslash K$ and such that the pluripolar hulls of their graphs are infinitely sheeted. The paper is motivated by a recent paper of Poletsky and Wiegerinck.
\end{abstract}

1. Introduction. The paper is motivated by a recent paper of E. Poletsky and J. Wiegerinck (see [8]).

Let $E \subset \mathbb{C}^{n}$ be any subset. We say that $E$ is pluripolar if for any $z \in E$ there exist a connected neighborhood $U_{z}$ of $z$ and a plurisubharmonic function $u_{z}$ defined on $U_{z}, u_{z} \not \equiv-\infty$, such that

$$
E \cap U_{z} \subset\left\{w \in U_{z}: u_{z}(w)=-\infty\right\} .
$$

By Josefson's theorem (see [5]) the set $E$ is pluripolar if and only if there exists a plurisubharmonic function $u, u \not \equiv-\infty$, defined on $\mathbb{C}^{n}$ such that $E \subset\left\{w \in \mathbb{C}^{n}: u(w)=-\infty\right\}$. It is well known (see e.g. [6]) that $\max \left\{\log \left|f_{1}\right|, \ldots, \log \left|f_{m}\right|\right\}$ is a plurisubharmonic function for any holomorphic functions $f_{1}, \ldots, f_{m}$. Therefore, the pluripolar sets are a generalization of analytic ones. So, it seems natural to study, with regard to pluripolar sets, problems similar to those studied in the case of analytic sets. In particular, we may study extension type problems. For this we use the notion of a pluripolar hull.

We denote by $\operatorname{PSH}(\Omega)$ the set of all plurisubharmonic functions defined on an open set $\Omega \subset \mathbb{C}^{n}$. For a pluripolar set $E \subset \Omega$ we define its pluripolar

2000 Mathematics Subject Classification: Primary 32D15, 32U30; Secondary 31C10, $30 \mathrm{C} 85$.

Key words and phrases: pluripolar hull, harmonic measure, logarithmic capacity.

Research supported by KBN grants No. 1 PO3A 00528 and PO3A 047 22. Research of the first author also supported by Krzyżanowski Fund and of the third by Rector's Scholarship Fund at the Jagiellonian University. 
hull as

$$
E_{\Omega}^{*}=\bigcap_{h \in \operatorname{PSH}(\Omega),\left.h\right|_{E} \equiv-\infty}\{w \in \Omega: h(w)=-\infty\} .
$$

For properties of the hull see e.g. [13, 7],

Let $D$ be a domain in $\mathbb{C}$ and let $f$ be a holomorphic function on $D$. For any subset $A \subset D$ we denote the graph of $f$ over $A$ by

$$
\Gamma_{f}(A)=\{(z, f(z)): z \in A\} .
$$

Note that if $A$ is a non-polar subset of $D$ then $\left(\Gamma_{f}(A)\right)_{\mathbb{C}^{2}}^{*}=\left(\Gamma_{f}(D)\right)_{\mathbb{C}^{2}}^{*}$. Our aim is to find conditions on $K$ which would imply the existence of a bounded holomorphic function on $\mathbb{C} \backslash K$ such that $\mathbb{C} \backslash K$ is the domain of existence of the function and the pluripolar hull of its graph is at least two-sheeted. It follows from [3] that there are no holomorphic functions on $\mathbb{C} \backslash K$ whose graphs have multiple sheeted pluripolar hulls if the set $K$ is polar. In [8] the authors constructed a Cantor type set $K$ on the real line and a holomorphic function $f$ with the domain of existence $\mathbb{C} \backslash K$ such that $\left(\Gamma_{f}(\mathbb{C} \backslash K)\right)_{\mathbb{C}^{2}}^{*}$ is double sheeted over $\mathbb{C} \backslash K$.

Let us fix some notations which will be used throughout the paper. We put $\mathbb{D}_{R}=\{z \in \mathbb{C}:|z|<R\}$, where $R>0$. We denote by $\mathbb{D}$ the unit disc $\mathbb{D}_{1}$ and $\mathbb{D}^{\mathrm{e}}:=\mathbb{C} \backslash \overline{\mathbb{D}}$. We also denote by $\mathbb{D}(p, r)$ the open disc with center at $p \in \mathbb{C}$ and radius $r>0$ and $H_{-}:=\{z \in \mathbb{C}: \operatorname{Im} z<0\}$. Let "cap" denote the logarithmic capacity and let $\omega(z, A, D)$ denote the harmonic measure for the domain $D \subset \mathbb{C}$ of the Borel set $A \subset \partial D, z \in D$ (for definition and basic properties see e.g. [9]). Let $m$ denote the normalized Lebesgue measure on the circle $\partial \mathbb{D}(p, r)$ (i.e. $m(\partial \mathbb{D}(p, r))=r)$ or the Lebesgue measure on $\mathbb{R}$.

The main result of the paper is the following.

Theorem 1. Let I denote the unit circle in $\mathbb{C}$ or an open interval $(a, b)$, where $-\infty<a<b<\infty$. Let $K$ be a compact subset of $I$ such that $\operatorname{cap}(I \backslash K)<\operatorname{cap}(I)$. Then the following conditions are equivalent:

(1) $m(K \cap U)>0$ for any $z \in K$ and for any neighborhood $U$ of $z$.

(2) There exists a bounded holomorphic function with the domain of existence equal to $\mathbb{C} \backslash K$.

(3) There exist a bounded holomorphic function $f$ whose domain of existence is $\mathbb{C} \backslash K$ and a set $K_{0} \subset K$ with $m\left(K_{0}\right)>0$ such that the non-tangential limits for $\left.f\right|_{\mathbb{D}}$ (respectively, $\left.\left.f\right|_{H_{-}}\right)$exist on $K_{0}$ and for any $z \in(\mathbb{C} \backslash K) \cup K_{0}$ the set $\left\{w \in \mathbb{C}:(z, w) \in\left(\Gamma_{f}(\mathbb{C} \backslash K)\right)_{\mathbb{C}^{2}}^{*}\right.$ is infinite.

It will follow from the proof that in the case $K \neq \partial \mathbb{D}$ we shall be able to choose $f$ so that the pluripolar hull $\left(\Gamma_{f}(\mathbb{C} \backslash K)\right)_{\mathbb{C}^{2}}^{*}$ contains the set 


$$
\left\{\left(z, e^{2 k} f(z)\right): z \in \mathbb{C} \backslash K\right\} \cup\left\{\left(z, e^{2 k} f^{*}(z)\right): z \in K_{0}\right\}
$$

for any $k \in \mathbb{Z}$, where $f^{*}(z)$ denotes the non-tangential limit of $\left.f\right|_{\mathbb{D}}$ (respectively, $\left.\left.f\right|_{H_{-}}\right)$at $z \in K_{0}$.

The condition $\operatorname{cap}(I \backslash K)<\operatorname{cap}(I)$ implies, in particular, that $K$ is not empty, so condition (1) is not trivial.

It will follow from the proof that the assumption $\operatorname{cap}(I \backslash K)<\operatorname{cap}(I)$ may be replaced with some condition on the existence of a set $G$ such that $I \backslash G$ is thin at each point of a set of positive measure (cf. Theorem 7).

It is worth noting that the existence of non-constant holomorphic mappings on $\mathbb{C} \backslash K$ implies that $K$ is of positive analytic capacity (see e.g. [12]).

The implication $(2) \Rightarrow(1)$ in Theorem 1 is well known (see e.g. [1, Appendix A 1.4]). The implication $(3) \Rightarrow(2)$ is trivial. So, the main result of the paper is the implication $(1) \Rightarrow(3)$.

Note that in the case $K=\partial \mathbb{D}$ the Blaschke products from [14] help us to produce examples similar to that from (3) - the functions have, however, a little weaker properties than the ones claimed in Theorem 1 . In fact, one may take the function $f$ defined as $f(z):=\log (2+z) B(z)$ for $z \in \mathbb{D}$ and $f(z):=f(1 / z), z \in \mathbb{D}^{\mathrm{e}}$. Then the pluripolar hull $\left(\Gamma_{f}(\mathbb{C} \backslash \partial \mathbb{D})\right)_{\mathbb{C}^{2}}^{*}$ is generically (with the possible exception of at most countably many points in $\mathbb{C} \backslash \partial \mathbb{D}$ ) infinitely sheeted. The methods from [10] could also help to exhibit a set $K_{0}$ as in Theorem 1.

In our paper except for pluripolar hulls we use mainly results and notions from potential theory on the complex plane. Apart from the notions of logarithmic capacity and harmonic measure we use, among others, the notions of logarithmic potential, equilibrium measure and Green function. The book [9] may serve as a good reference for the notions from potential theory used in this paper.

We mostly prove our results for subsets of $\partial \mathbb{D}$. The case of subsets of $\mathbb{R}$, when handled along the same lines, is sometimes only sketched or some details are omitted.

\section{Useful results on the Green function and harmonic measure.} Let $K \subset \mathbb{C}$ be a non-polar compact set in the complex plane. We define its Green function as

$$
g_{K}(z)=p_{\mu}(z)-\log \operatorname{cap}(K),
$$

where $\mu$ is the equilibrium measure of $K$ and $p_{\mu}(z)=\int_{K} \log |z-w| d \mu(w)$ (for properties of the Green function see [9]). We extend the definition of the Green function to bounded non-polar $F_{\sigma}$-sets. Assume that $F=\bigcup K_{j}$, where $K_{j}$ is an increasing sequence of non-polar compact sets. Then $\left\{g_{K_{j}}\right\}$ is a decreasing sequence of subharmonic functions. Put $g_{F}(z)=\lim _{j \rightarrow \infty} g_{K_{j}}(z)$. 
The following properties are well known:

- $g_{F} \in \mathrm{SH}(\mathbb{C}) \cap(\mathbb{C} \backslash \bar{F})$;

- $g_{F} \geq 0$ on $\mathbb{C}$;

- $g_{F}=0$ on $F \backslash E$, where $E$ is a Borel polar set;

- if $F \subset \partial \mathbb{D}$ then $g_{F}(0)=-\log \operatorname{cap}(F)$.

Lemma 2. Let $F \subset \partial \mathbb{D}$ be an $F_{\sigma}$-set such that $\operatorname{cap}(F)<1$. Then there exists a Borel set $L \subset \partial \mathbb{D} \backslash F$ of positive measure such that $F$ is thin at every point of $L$.

Let $F \subset(a, b)$, where $-\infty<a<b<\infty$, be an $F_{\sigma}$-set such that cap $(F)<$ $\operatorname{cap}((a, b))=(b-a) / 4$. Then there exists a subset $L$ of $(a, b) \backslash F$ of positive measure such that $F$ is thin at every point of $L$.

Proof. Without loss of generality we may assume in both cases that $F$ is not polar.

Assume that $F \subset \partial \mathbb{D}$. The properties of $g_{F}$ imply that

$$
0<-\log \operatorname{cap}(F)=g_{F}(0)=\frac{1}{2 \pi} \int_{0}^{2 \pi} g_{F}\left(e^{i \theta}\right) d \theta .
$$

Note that the Borel set $L:=\left\{z \in \partial \mathbb{D} \backslash F: g_{F}(z)>0\right\} \subset \partial \mathbb{D} \backslash F$ is of positive measure. We claim that $F$ is thin at every point of $L$. Indeed, $g_{F}=0$ on $F \backslash E$, where $E \subset F$ is a Borel polar set. Then $F \backslash E$ and $E$ (and, therefore, $F=(F \backslash E) \cup E))$ are thin at every point of $L$.

In the case $F \subset(a, b)$, we may assume that $a=-1$ and $b=1$. Put $I=[-1,1]$. Then the function

$$
u(z)=g_{F}(z)-g_{I}(z), \quad u(\infty)=\log \frac{b-a}{4 \operatorname{cap} F},
$$

is a positive harmonic bounded function on $\widehat{\mathbb{C}} \backslash I$. The function $v(\zeta)=$ $u\left(\frac{1}{2}(\zeta+1 / \zeta)\right)$ is harmonic in the unit disc and $\lim _{r \rightarrow 1^{-}} v\left(r e^{i t}\right)=v^{*}\left(e^{i t}\right)=$ $g_{F}(\cos t)$ for almost all $t \in[0,2 \pi)$. Therefore,

$$
0<u(\infty)=v(0)=\frac{1}{2 \pi} \int_{0}^{2 \pi} v^{*}\left(e^{i t}\right) d t=\frac{1}{\pi} \int_{0}^{\pi} g_{F}(\cos t) d t .
$$

Hence, the set $L=\left\{z \in(a, b) \backslash F: g_{F}(z)>0\right\}$ has positive Lebesgue measure and so, as in the previous case, $F$ is thin at every point of $L$.

Now we are going to give some estimate of the harmonic measure at thin points. In the context of pluripolar hulls it was proved in [2]. In [4] the authors observed that from the proof it follows that the estimate holds in some fine neighborhood of a thin point. So, let $A \subset \mathbb{C}$ be a set thin at a point $p \in \mathbb{C}$. It is well known that there exists a sequence $\varrho_{k} \searrow 0$ such that $A \cap \partial \mathbb{D}\left(p, \varrho_{k}\right)=\emptyset($ see $[9$, Theorem 5.4.2]). 
Lemma 3. Let $A \subset \mathbb{C}$ be a set thin at a point $p \in \mathbb{C}$. Then for any $\varepsilon>0$ there exist $\varrho \in(0, \varepsilon), r \in(0, \varrho)$, and an open set $U_{1} \supset(A \cap \mathbb{D}(p, \varrho)) \backslash\{p\}$ such that

- $A \cap \partial \mathbb{D}(p, \varrho)=\emptyset, \partial \mathbb{D}(p, r) \cap U_{1}=\emptyset$;

- $U_{1}$ is thin at $p$;

- $\omega(z, J, \mathbb{D}(p, \varrho) \backslash F) \geq m(J) / \varrho-\varepsilon$ for any $z \in \overline{\mathbb{D}(p, r)} \backslash U_{1}$, where $J$ is any closed subarc of $\partial \mathbb{D}(p, \varrho)$ and $F$ is any closed (in $\mathbb{C})$ subset of $A$.

Proof. Note that if $p \notin \overline{A \backslash\{p\}}$ then the assertion is trivial (recall that $\omega(0, J, \mathbb{D}(p, \varrho))=m(J) / \varrho$, take $r$ sufficiently small and $\left.U_{1}=\emptyset\right)$. So, we may assume that $p \in \overline{A \backslash\{p\}}$.

Let $\mathcal{U} \in \mathrm{SH}(\mathbb{C})$ be an entire subharmonic function such that

$$
\lim _{z \rightarrow p, z \in A} \mathcal{U}(z)=-\infty
$$

and $\mathcal{U}(p)>-\infty$. By adding a constant to $\mathcal{U}$ we may assume that $\mathcal{U}<0$ on $\overline{D(p, \varrho)}$. Multiplying $\mathcal{U}$ by a positive constant we may assume that $\mathcal{U}(p)>-\varepsilon / 2$. Taking $0<\varrho<\varepsilon$ small enough, we may assume that $A \cap \partial \mathbb{D}(p, \varrho)=\emptyset$ and $\mathcal{U}(z)<-1$ on $(A \cap \overline{\mathbb{D}(p, \varrho)}) \backslash\{p\}$. Then for any $\operatorname{arc} J \subset \partial \mathbb{D}(p, \varrho)$ and any closed $F \subset A$,

$$
\omega(z, J, \mathbb{D}(p, \varrho) \backslash F) \geq \omega(z, J, \mathbb{D}(p, \varrho))+\mathcal{U}(z), \quad z \in \mathbb{D}(p, \varrho) \backslash F .
$$

Put $U_{1}:=\{z \in \mathbb{D}(p, \varrho): \mathcal{U}(z)<-\varepsilon / 2\}$. Then $U_{1}$ is open, $U_{1} \supset$ $(A \cap \mathbb{D}(p, \varrho)) \backslash\{p\}$, and since $\mathcal{U}(p)>-\varepsilon / 2$ the set $U_{1}$ is thin at $p$, with $p \in \overline{U_{1} \backslash\{p\}}$. We know that $\omega(p, J, \mathbb{D}(p, \varrho))=m(J) / \varrho$. Hence, we may choose $0<r<\varrho$ with $U_{1} \cap \partial \mathbb{D}(p, r)=\emptyset$ and $\omega(z, J, \mathbb{D}(p, \varrho))>m(J) / \varrho-\varepsilon / 2$ for $z \in \overline{\mathbb{D}(p, r)}$. Then by (1),

$$
\omega(z, J, \mathbb{D}(p, \varrho) \backslash F) \geq \frac{m(J)}{\varrho}-\varepsilon, \quad z \in \overline{\mathbb{D}(p, r)} \backslash U_{1} .
$$

Now we study the case when $A \subset \partial \mathbb{D}$ is thin at $p \in \partial \mathbb{D}$. First note that for every $\varrho$ with $0<\varrho<1$ there exists a closed subarc $J$ of the $\operatorname{arc} \mathbb{D} \cap \partial \mathbb{D}(p, \varrho)$ (respectively, $\mathbb{D}^{\mathrm{e}} \cap \partial \mathbb{D}(p, \varrho)$ ) with $m(J) \geq \varrho / 3$. So, from Lemma 3 we get the following.

Corollary 4. Let $A \subset \partial \mathbb{D}$ be a set thin at a point $p \in \partial \mathbb{D}$. Then for any $\varepsilon>0$ there exist $\varrho \in(0, \varepsilon), r \in(0, \varrho)$, and an open set $U_{1} \supset A \cap \mathbb{D}(p, \varrho) \backslash\{p\}$ such that $A \cap \partial \mathbb{D}(p, \varrho)=\emptyset, \partial \mathbb{D}(p, r) \cap U_{1}=\emptyset, U_{1}$ is thin at $p$, and

$$
\omega(z, J, \mathbb{D}(p, \varrho) \backslash F) \geq 1 / 4, \quad z \in \overline{\mathbb{D}(p, r)} \backslash U_{1},
$$

where $J$ is any closed subarc of the arc $\mathbb{D} \cap \partial \mathbb{D}(p, \varrho)$ (respectively, $\mathbb{D}^{\mathrm{e}} \cap$ $\partial \mathbb{D}(p, \varrho))$ such that $m(J) \geq \varrho / 3$ and $F$ is any closed $($ in $\mathbb{C})$ subset of $A$. 


\section{Construction of a decreasing sequence of open sets}

Proposition 5. Let I denote either the unit circle $\partial \mathbb{D}$ or an interval $(a, b)$, where $-\infty<a<b<\infty$. Let $K \subset I$ be a non-empty compact set such that $m(K \cap U)>0$ for any open set $U$ such tha $U \cap K \neq \emptyset$. Then there is a decreasing sequence $\left(G_{n}\right)_{n=1}^{\infty}$ of open sets in I with the following properties:

- $0<m(U \cap G)<m(G)$ for any open set $U \subset I$ such that $U \cap K \neq \emptyset$, where $G=\bigcap G_{n}$;

- $\bar{G}_{n}=K$;

- there exists a Borel set $L \subset G$ of positive measure such that $I \backslash G$ is thin at every point of $L$.

It follows from Lemma 2 that for the existence of $L$ as in the proposition it is sufficient to construct $G$ so that $\operatorname{cap}(I \backslash G)<\operatorname{cap}(I)$.

Denote by $\mathcal{C}_{1}$ the family of non-trivial connected components of $K$ and by $\mathcal{C}_{2}$ the family of one-point connected components of $K$. Then $\mathcal{C}_{1}$ is at most countable (it may be empty). It will follow from the proof that in the case $\mathcal{C}_{1}=\emptyset$ the equality $G=K$ holds.

LEMma 6. Let $I$ be either an open arc in $\partial \mathbb{D}$ (we allow $I$ to be equal to $\partial \mathbb{D})$ or an interval $(a, b)$, where $-\infty<a<b<\infty$, and $\varepsilon>0$. Then there is a decreasing sequence $\left(G_{n}\right)$ of open sets in $I$, dense in $I$, such that

$$
0<m(J \cap G)<m(J)
$$

for any non-trivial open arc $J \subset I$, where $G:=\bigcap_{n \geq 1} G_{n}$ and

$$
\operatorname{cap}(I \backslash G)<\varepsilon .
$$

Assume for a while that the lemma holds. Then we may complete the proof of the proposition.

Proof of Proposition 5. We define $G_{n}$ to be the union of $G_{n}^{\text {int } P}$ over all $P \in \mathcal{C}_{1}\left(G_{n}^{\text {int } P}\right.$ is the set constructed as in the lemma with $\left.I=\operatorname{int} P\right)$ and $\left\{z \in I: \operatorname{dist}\left(z, \cup \mathcal{C}_{2}\right)<1 / n\right\}$. Note that if $\mathcal{C}_{1}$ is empty then $G=K$. For the last statement it suffices to use Lemma 2 (in the case $\mathcal{C}_{1} \neq \emptyset$ we need to use, additionally, the previous lemma for one $\left.P \in \mathcal{C}_{1}\right)$.

Therefore, it is sufficient to show the above lemma.

Proof of Lemma 6. For an arbitrary non-trivial closed arc (interval) $P$ we make the following construction of Cantor-type sets (we follow the notation from [9]). Below, we use the word arc (or subarc) for interval (subinterval). For a sequence $\mathbf{s}:=\left(s_{n}\right)_{n=1}^{\infty}$ of numbers such that $0<s_{n}<1$ we define $C\left(s_{1}, P\right)$ to be the set obtained by removing from $P$ the concentric (in the case $I=\partial \mathbb{D}$ the choice is arbitrary) arc whose length is an $s_{1}$ fraction of the length of the whole arc. At the $n$th stage let $C\left(s_{1}, \ldots, s_{n}, P\right)$ be the set obtained by removing from each connected component of $C\left(s_{1}, \ldots, s_{n-1}, P\right)$ 
the open concentric arc whose length is an $s_{n}$ fraction of the whole component. We define

$$
C(\mathbf{s}, P):=\bigcap_{n=1}^{\infty} C\left(s_{1}, \ldots, s_{n}, P\right) .
$$

Recall that (see e.g. [9]) the Lebesgue measure of $C(\mathbf{s}, P)$ equals $m(P) t(\mathbf{s}):=$ $m(P) \prod_{n=1}^{\infty}\left(1-s_{n}\right)$. Below we choose a sequence of sequences $\mathbf{s}$ satisfying additional assumptions. For $\theta \in(0,1)$ we define $\mathbf{s}(\theta)=\left(s_{1}(\theta), s_{2}(\theta), \ldots\right)$ as follows:

$$
s_{j}(\theta):= \begin{cases}3^{-j} & \text { for } j \neq 2 \\ \theta & \text { for } j=2 .\end{cases}
$$

Note that $t(\theta):=t(\mathbf{s}(\theta))=C_{0}(1-\theta)>0$ for some constant $C_{0} \in(0,1)$.

Below we choose a sequence $\left(\theta_{N}\right)$ of numbers from $(0,1)$ such that $0<\theta_{1}<\theta_{2}<\cdots<1, \lim _{N \rightarrow \infty} \theta_{N}=1$ and $\prod_{N=1}^{\infty}\left(1-t^{N}\right)>0$, where $t^{N}:=t\left(\theta_{N}\right)$.

For a relatively open (in $I$ ) subset $\Omega$ and $\theta \in(0,1)$ we construct an open set $\Omega(\theta)$ as follows. Let us represent $\Omega$ as the union of at most countably many open, disjoint arcs $I_{j}$-these are all connected components of $\Omega$. Then we define

$$
\Omega(\theta):=\bigcup_{j}\left(I_{j} \backslash C\left(\mathbf{s}(\theta), \bar{I}_{j}\right)\right)=\bigcup_{j}\left(\bar{I}_{j} \backslash C\left(\mathbf{s}(\theta), \bar{I}_{j}\right)\right) .
$$

Note that $m(\Omega(\theta))=m(\Omega)(1-t(\theta))$ and $\Omega(\theta) \nearrow_{\theta \rightarrow 1^{-}} \Omega \backslash A$, where $A$ is at most countable.

Now we come back to the proof of the lemma. We make an inductive construction based on a proper choice of a sequence $\left(\theta_{N}\right)$ with the above mentioned properties. We define, simultaneously, two sequences of sets.

We start with $G_{0}:=I, K_{0}:=\emptyset$. Then we define $K_{1}:=C\left(\mathbf{s}\left(\theta_{1}\right), \bar{I}\right)$, $G_{1}=I\left(\theta_{1}\right)=I \backslash C\left(\mathbf{s}\left(\theta_{1}\right), \bar{I}\right)$, where $\theta_{1} \in(0,1)$ is chosen so that $\operatorname{cap}\left(I \backslash G_{1}\right)$ $<\varepsilon / 2$. Then we define inductively sets $G_{N+1}, K_{N+1}$ and a number $\theta_{N}$ as follows. If $G_{N}, K_{N}$ and the numbers $0<\theta_{1}<\cdots<\theta_{N}<1$ are already constructed so that $\operatorname{cap}\left(I \backslash G_{N}\right)<\varepsilon\left(1 / 2+\cdots+1 / 2^{N}\right)$, we define $G_{N+1}:=$ $G_{N}\left(\theta_{N+1}\right), K_{N+1}:=K_{N+1}\left(\theta_{N+1}\right):=\bigcup_{j} C\left(\mathbf{s}\left(\theta_{N+1}\right), \bar{I}_{j}^{N}\right)$, where $\left(I_{j}^{N}\right)_{j}$ are the connected components of $G_{N}$, and $1>\theta_{N+1}>\theta_{N}$ is chosen so that $\operatorname{cap}\left(I \backslash G_{N+1}\right)=\operatorname{cap}\left(\left(I \backslash G_{N}\right) \cup K_{N+1}\right)<\varepsilon\left(1 / 2+\cdots+1 / 2^{N+1}\right)$; note that $K_{N+1}\left(\theta_{N+1}\right) \searrow B$ as $\theta_{N+1} \rightarrow 1^{-}$, where $B$ is at most countable, so when $\theta_{N+1} \in\left(\theta_{N}, 1\right)$ is sufficiently close to $1, \operatorname{cap}\left(K_{N+1}\left(\theta_{N+1}\right)\right)$ may be chosen to be arbitrarily small. It is no problem to choose $\left(\theta_{N}\right)_{N}$ so that $\lim _{N \rightarrow \infty} \theta_{N}=1$ and $\prod_{N=1}^{\infty}\left(1-t^{N}\right)>0$.

The assumption $s_{1}(\theta)=1 / 3$ implies that for any $N$ the set $\bar{I} \backslash G_{N}$ is totally disconnected (and certainly compact). Moreover, the inequality 
$m\left(I_{j}^{N}\right) \leq 1 / 3^{N}$ holds for all possible $j, N$, where $G_{N}=\bigcup_{j} I_{j}^{N}$ and $\left(I_{j}^{N}\right)_{j}$ is the family of connected components of $G_{N}$.

Now we prove that for a non-trivial open arc $J \subset I$ the inequalities $0<m(J \cap G)<m(J)$ hold.

Fix a non-trivial open arc $J \subset I$. Then there are $N$ and $j$ such that $\bar{I}_{j}^{N} \subset J$. Hence

$$
m(J \cap G) \geq m\left(I_{j}^{N} \cap G\right)=m\left(I_{j}^{N}\right) \prod_{k=N+1}^{\infty}\left(1-t^{k}\right)>0 .
$$

On the other hand, the inequality $m(J \cap G)<m(J)$ is equivalent to the inequality $m(J \backslash G)>0$. But $m(J \backslash G) \geq m\left(I_{j}^{N} \backslash G\right) \geq m\left(I_{j}^{N} \backslash G_{N+1}\right)=m\left(\bar{I}_{j}^{N} \backslash G_{N+1}\right)=m\left(I_{j}^{N}\right) t^{N+1}>0$.

4. Construction of holomorphic functions. In the whole section we fix a non-empty compact set $K \subset \partial \mathbb{D}$ and a decreasing sequence of open sets $\left(G_{n}\right)_{n \in \mathbb{N}} \subset \partial \mathbb{D}$ such that $\bigcap_{n=1}^{\infty} \bar{G}_{n}=K$. We are going to construct a holomorphic function $f$ on $\mathbb{C} \backslash K$ with special properties.

Put $G=\bigcap G_{n}$. Let $u_{n}$ (resp. $u$ ) denote the solution of the Dirichlet problem on $\mathbb{D}$ with the boundary data $\chi_{G_{n}}\left(\right.$ resp. $\left.\chi_{G}\right)$. Let $v_{n}$ (resp. $v$ ) denote the conjugate to $u_{n}($ resp. $u)$ with $v_{n}(0)=0($ resp. $v(0)=0)$. Put $\varphi_{n}=u_{n}+i v_{n}$ and $\varphi=u+i v$.

First recall the following result related to the solution of the Dirichlet problem on the unit disc.

Theorem 7 (see [9, Theorem 1.2.4], [11, Theorem IV.1]). Let $\psi \in$ $L^{1}(\partial \mathbb{D})$. Put

$$
u_{\psi}(z)=\frac{1}{2 \pi} \int_{0}^{2 \pi} \frac{1-|z|^{2}}{\left|e^{i \theta}-z\right|^{2}} \psi\left(e^{i \theta}\right) d \theta .
$$

Then

(a) $u_{\psi}$ is a harmonic function on $\mathbb{D}, \inf _{\partial \mathbb{D}} \psi \leq u_{\psi} \leq \sup _{\partial \mathbb{D}} \psi$;

(b) $u_{\psi}^{*}\left(e^{i \theta}\right)=\lim _{r \rightarrow 1} u_{\psi}\left(r e^{i \theta}\right)$ exists for almost all $\theta \in[0,2 \pi)$; moreover, $u_{\psi}^{*}=\psi$ a.e. on $\partial \mathbb{D}$

(c) if $\psi$ is continuous at $\zeta_{0} \in \partial \mathbb{D}$, then $u_{\psi}$ extends continuously to $\zeta_{0}$.

From Theorem 7 we obtain

$$
\varphi_{n}(z)=\frac{1}{2 \pi} \int_{0}^{2 \pi} \frac{e^{i \theta}+z}{e^{i \theta}-z} \chi_{G_{n}}\left(e^{i \theta}\right) d \theta .
$$

An analogous formula holds for $\varphi$. Note that $\operatorname{Re} \varphi_{n} \in C\left(\overline{\mathbb{D}} \backslash \partial G_{n}\right)$. Moreover, $\operatorname{Re} \varphi_{n}=1$ on $G_{n}$ and $\operatorname{Re} \varphi_{n}=0$ on $\partial \mathbb{D} \backslash \bar{G}_{n}$. Here the boundary of $G_{n}$ is taken with respect to the topology of $\partial \mathbb{D}$. 


\section{LEMMA 8.}

(a) $\varphi_{n}$ extends continuously to $\partial \mathbb{D} \backslash \partial G_{n}$.

(b) The sequence $\left\{\varphi_{n}\right\}_{n=1}^{\infty}$ tends to $\varphi$ locally uniformly on $\mathbb{D}$.

Proof. (a) Let $I \subset G_{n}$ be an open arc. Without loss of generality we may assume that $I=\left\{e^{i \theta}: 0<\theta<\theta_{I}\right\}$, where $\theta_{I} \in(0,2 \pi)$. Then

$$
\varphi_{n}(z)=\frac{1}{2 \pi} \int_{0}^{2 \pi} \frac{e^{i \theta}+z}{e^{i \theta}-z} \chi_{G_{n} \backslash I}\left(e^{i \theta}\right) d \theta+\frac{1}{2 \pi} \int_{0}^{2 \pi} \frac{e^{i \theta}+z}{e^{i \theta}-z} \chi_{I}\left(e^{i \theta}\right) d \theta=: I_{1}+I_{2} .
$$

Fix $\theta_{0} \in\left(0, \theta_{I}\right)$. Note that $I_{1}$ extends continuously to $e^{i \theta_{0}}$ and

$$
I_{2}=\frac{1}{\pi i} \log \left(e^{-\theta_{I} / 2} \frac{z-e^{i \theta}}{z-1}\right)
$$

where $\log :\{z \in \mathbb{C}: \operatorname{Im} z>0\} \rightarrow\{z \in \mathbb{C}: 0<\operatorname{Im} z<\pi\}$ is such that $\log i=\pi i / 2$, so it also extends continuously to $e^{i \theta_{0}}$.

(b) Fix $r \in(0,1)$. Then for any $z \in \mathbb{D}_{r}$ we have

$$
\left|\varphi_{n}(z)-\varphi(z)\right|=\left|\frac{1}{2 \pi} \int_{0}^{2 \pi} \frac{e^{i \theta}+z}{e^{i \theta}-z} \chi_{G_{n} \backslash G}\left(e^{i \theta}\right) d \theta\right| \leq \frac{2}{1-r} m\left(G_{n} \backslash G\right) .
$$

The last expression tends to 0 as $n \rightarrow \infty$.

Put $f_{n}=\exp \left(\varphi_{n}\right)$ and $f=\exp (\varphi)$. Note that $f_{n}$ extends to a function from $\mathcal{O}\left(\mathbb{C} \backslash \bar{G}_{n}\right)$, denoted also by $f_{n}$. Even more, the extension is defined by the formula $f_{n}(z)=1 / \overline{f_{n}(1 / \bar{z})}, z \in \mathbb{D}^{\mathrm{e}}$. For $z \in \mathbb{D}^{\mathrm{e}}$ we also put $f(z)=$ $1 / \overline{f(1 / \bar{z})}$. From Lemma 8 we see that the sequence $\left(f_{n}\right)_{n=1}^{\infty}$ is convergent to $f$ locally uniformly on $\mathbb{D} \cup \mathbb{D}^{\mathrm{e}}$.

Note that $e^{-1}<\left|f_{n}\right|<e$ on $\mathbb{C} \backslash \bar{G}_{n}$ and $e^{-1}<|f|<e$ on $\mathbb{C} \backslash K$. Note also that $\lim _{r \rightarrow 1^{-}}|f(r z)|=e$ for almost all $z \in G$ (consequently, $\lim _{r \rightarrow 1^{+}}|f(r z)|=e^{-1}$ for almost all $\left.z \in G\right)$ and $\lim _{r \rightarrow 1^{-}}|f(r z)|=1$ for almost all $z \in \partial \mathbb{D} \backslash G$ (hence $\lim _{r \rightarrow 1^{+}}|f(r z)|=1$ for almost all $z \in \partial \mathbb{D} \backslash G$ ).

It is easy to see that if $0<m(U \cap G)<m(U)$ for any non-empty open set $U$ such that $U \cap K \neq \emptyset$ then $\mathbb{C} \backslash K$ is the domain of existence of $f$.

5. Proof of Theorem 1. By Lemma 2 to prove Theorem 1 it suffices to prove the following result. We shall prove it for $K \subset \partial \mathbb{D}$. The proof for $K \subset \mathbb{R}$ follows exactly the same lines, so we omit it.

THEOREM 9. Let I denote either the unit circle or an interval $(a, b) \subset \mathbb{R}$, where $a<b$. Let $K \subset I$ be a compact subset of $I$ for which there exists $a$ decreasing sequence $\left\{G_{n}\right\}$ of open subsets of $I$ with the following properties:

- $0<m(G \cap U)<m(U)$ for every open set $U \subset I$ with $U \cap K \neq \emptyset$, where $G:=\bigcap_{n=1}^{\infty} G_{n}$;

- $K=\bigcap_{n=1}^{\infty} \bar{G}_{n}$; 
- there exists a set $L \subset G$ such that $m(L)>0$ and $I \backslash G$ is thin at every point of $L$ (which holds, for instance, when $\operatorname{cap}(I \backslash G)<\operatorname{cap} I-$ see Lemma 2).

Put

$$
f(z):=f_{K}(z):=\exp \frac{1}{2 \pi} \int_{0}^{2 \pi} \frac{e^{i t}+z}{e^{i t}-z} \chi_{G}\left(e^{i t}\right) d t,
$$

respectively,

$$
f(z):=f_{K}(z):=\exp \frac{1}{\pi i} \int_{-\infty}^{\infty} \frac{\chi_{G}(t)}{t-z} d t,
$$

for $z \in \mathbb{C} \backslash K$. Then $f_{K}$ is a bounded holomorphic function on $\mathbb{C} \backslash K$ whose maximal domain of existence is $\mathbb{C} \backslash K$ and $1 / e<\left|f_{K}\right|<e$ on $\mathbb{C} \backslash K$.

In the case $K \neq \partial \mathbb{D}$ there exists a set $K_{0} \subset K$ with $m\left(K_{0}\right)>0$ such that the non-tangential limits of $\left.f\right|_{\mathbb{D}}$ (respectively, $\left.\left.f\right|_{H_{-}}\right)$exist on $K_{0}$ and the pluripolar hull $\left(\Gamma_{f}(\mathbb{C} \backslash K)\right)_{\mathbb{C}^{2}}^{*}$ contains the set

$$
\left\{\left(z, e^{2 k} f(z)\right): z \in \mathbb{C} \backslash K\right\} \cup\left\{\left(z, e^{2 k} f^{*}(z)\right): z \in K_{0}\right\}
$$

for any $k \in \mathbb{Z}$, where $f^{*}(z)$ denotes the non-tangential limit of $\left.f\right|_{\mathbb{D}}$ (respectively, $\left.\left.f\right|_{H_{-}}\right)$at $z \in K_{0}$.

In the case $K=\partial \mathbb{D}$ we define $K^{1}:=\partial \mathbb{D} \cap \bar{H}_{-}, K^{2}:=\partial \mathbb{D} \cap \bar{H}_{+}$and $f:=f_{1}+f_{2}:=f_{K^{1}}+f_{K^{2}}$. Then $f$ is a bounded holomorphic function on $\mathbb{C} \backslash \partial \mathbb{D}$ whose maximal domain of existence is $\mathbb{C} \backslash \partial \mathbb{D}$ and there exist sets $K_{0}^{1} \subset K^{1}, K_{0}^{2} \subset K^{2}$ with $m\left(K_{0}^{j}\right)>0$ such that the non-tangential limits of $\left.f_{j}\right|_{\mathbb{D}}$ exist on $K_{0}^{j}$ and the pluripolar hull $\left(\Gamma_{f}(\mathbb{C} \backslash \partial \mathbb{D})\right)_{\mathbb{C}^{2}}^{*}$ contains the sets

$$
\left\{\left(z, e^{2 k} f_{1}(z)+e^{-2 k-2} f_{2}(z)\right): z \in \mathbb{D}\right\} \cup\left\{\left(z, e^{2 k} f_{1}(z)+e^{-2 k} f_{2}(z)\right): z \in \mathbb{D}^{\mathrm{e}}\right\}
$$

and

$$
\left\{\left(z, e^{2 k} f_{1}^{*}(z)+e^{-2 k-2} f_{2}^{*}(z)\right): z \in K_{0}^{1} \cup K_{0}^{2}\right\}
$$

for any $k \in \mathbb{Z}$.

Proof. As already mentioned, we restrict ourselves to the case $K \subset \partial \mathbb{D}$. Recall that $f=f_{K}$ is constructed in Section 4. Put

$$
K_{0}=\left\{a \in L:\left.f\right|_{\mathbb{D}} \text { has the angular limit value } f^{*}(a) \text { at } a\right\} .
$$

By the Fatou theorem the set $K_{0}$ has positive Lebesgue measure.

Now we are left with the proof of the properties of pluripolar hulls. Let us start with the case $K \neq \partial \mathbb{D}$. In particular, $\mathbb{C} \backslash K$ is connected. Let $p \in \operatorname{PSH}\left(\mathbb{C}^{2}\right)$ be such that

$$
p(z, f(z))=-\infty \quad \text { for any } z \in \mathbb{C} \backslash K .
$$


We need to show that

$$
p\left(z, e^{2 k} f(z)\right)=-\infty \quad \text { for any } z \in(\mathbb{C} \backslash K) \cup K_{0}, k \in \mathbb{Z},
$$

where we put $f(a):=f^{*}(a)$ for $a \in K_{0}$.

To prove (10) it is sufficient to show that

$$
p\left(z, F_{k}(z)\right)=-\infty \quad \text { for any } z \in \mathbb{D} \cup K_{0} \cup \mathbb{D}^{\mathrm{e}}, k \in \mathbb{Z},
$$

where $F_{k}$ is given by

$$
F_{k}(z)= \begin{cases}e^{2 k-2} f(z), & z \in \mathbb{D} \\ e^{2 k-2} f^{*}(z), & z \in K_{0} \\ e^{2 k} f(z), & z \in \mathbb{D}^{\mathrm{e}}\end{cases}
$$

We shall argue by induction with respect to $k$.

First let us show (11) for $k=0$. By the assumption $p(z, f(z))=-\infty$ for $z \in \mathbb{D}^{\mathrm{e}}$. Let $c$ be a fixed point of $K_{0}$. We now apply Corollary 4 to get

$$
\omega\left(z, J,(\mathbb{D}(c, \varrho) \backslash \partial \mathbb{D}) \cup\left(G_{n} \cap \partial \mathbb{D}\right)\right) \geq 1 / 4, \quad|z-c| \leq r_{1}, z \notin U_{1},
$$

where $J$ is some arc in $\mathbb{D}(c, \varrho) \cap \mathbb{D}^{\mathrm{e}}$ such that $m(J) \geq \varrho / 3$.

The functions

$$
F_{n, 0}(z)= \begin{cases}e^{-2} f_{n}(z), & z \in \mathbb{D} \cup G_{n} \\ f_{n}(z), & z \in \mathbb{D}^{\mathrm{e}}\end{cases}
$$

are holomorphic and uniformly bounded on $\mathbb{D} \cup G_{n} \cup \mathbb{D}^{\mathrm{e}}$, and $F_{n, 0} \rightarrow F_{0}$ locally uniformly on $\mathbb{D} \cup \mathbb{D}^{\mathrm{e}}$. Consider two cases:

$(\alpha) z_{0} \in \mathbb{D}$ and $\left|z_{0}-c\right|=r_{1}$;

( $\beta) z_{0}=c$.

In case $(\alpha)$ we put

$$
u_{n}(z)=p\left(z, F_{n, 0}(z)+F_{0}\left(z_{0}\right)-F_{n, 0}\left(z_{0}\right)\right)
$$

and

$$
M=\sup _{n \geq 1} \sup \left\{u_{n}(z): z \in(\overline{\mathbb{D}(c, \varrho)} \backslash \partial \mathbb{D}) \cup\left(\mathbb{D}(c, \varrho) \cap G_{n}\right)\right\} .
$$

Let $M_{n}:=\sup \left\{u_{n}(z): z \in J\right\}$. Then $M_{n} \rightarrow-\infty$ and the two constants theorem gives

$$
u_{n}(z) \leq M+\left(M_{n}-M\right) \omega\left(z, J,(\mathbb{D}(c, \varrho) \backslash \partial \mathbb{D}) \cup\left(\mathbb{D}(c, \varrho) \cap G_{n}\right)\right)
$$

for all $z \in \mathbb{D}\left(c, r_{1}\right) \backslash U_{1}, n \geq n_{0}$, where $n_{0}$ is so large that $M_{n}-M<0$ for $n \geq n_{0}$. It follows that

$$
p\left(z_{0}, F_{0}\left(z_{0}\right)\right)=u_{n}\left(z_{0}\right) \leq M+\left(M_{n}-M\right) \frac{1}{4} \rightarrow-\infty
$$

as $n \rightarrow \infty$. Since $z_{0}$ was chosen arbitrarily,

$$
p\left(z, F_{0}(z)\right)=p\left(z, e^{-2} f(z)\right)=-\infty
$$

when $|z-c|=r_{1}, z \in \mathbb{D}$. Consequently, $p\left(z, F_{0}(z)\right)=-\infty$ for all $z \in \mathbb{D} \cup \mathbb{D}^{\mathrm{e}}$. 
In case $(\beta)$ let $U_{1}$ be the set from Corollary 4 thin at the point $c$, and let $\left\{z_{k}\right\}$ be a sequence in $\mathbb{D} \backslash U_{1}$ such that $z_{k} \rightarrow c$. Let $\left\{n_{k}\right\}$ be a strictly increasing sequence of positive numbers such that

$$
\left|F_{n_{k}, 0}\left(z_{k}\right)-F_{0}\left(z_{k}\right)\right|<1 / k, \quad k=1,2, \ldots
$$

Put

$$
u_{k}(z)=p\left(z+c-z_{k}, F_{n_{k}, 0}(z)+F_{0}(c)-F_{n_{k}, 0}\left(z_{k}\right)\right)
$$

and

$$
\begin{aligned}
M & :=\sup _{k \geq 1} \sup \left\{u_{k}(z): z \in(\overline{\mathbb{D}(c, \varrho)} \backslash \partial \mathbb{D}) \cup\left(\mathbb{D}(c, \varrho) \cap G_{n_{k}}\right)\right\}, \\
M_{k} & :=\sup \left\{u_{k}(z): z \in J\right\} .
\end{aligned}
$$

It is clear that $M_{k} \rightarrow-\infty$ and proceeding as in case $(\alpha)$ we get

$$
p\left(c, F_{0}(c)\right)=u_{k}\left(z_{k}\right) \leq M+\left(M_{k}-M\right) \frac{1}{4}, \quad k \geq k_{0},
$$

which implies that $p\left(c, F_{0}(c)\right)=-\infty$. Since $c \in K_{0}$ was chosen arbitrarily, we have proved that (11) is true for $k=0$, i.e.,

$$
p\left(z, F_{0}(z)\right)=-\infty \quad \text { on } \mathbb{D} \cup K_{0} \cup \mathbb{D}^{\mathrm{e}} .
$$

Assume that

$$
p\left(z, F_{k}(z)\right)=-\infty \quad \text { on } \mathbb{D} \cup K_{0} \cup \mathbb{D}^{\mathrm{e}}
$$

for all $k \in \mathbb{Z}$ with $|k| \leq m$, where $m \geq 0$. It remains to show that (11) is true for all $k \in \mathbb{Z}$ with $|k|=m+1$.

First, let $k=m+1$. By the induction assumption

$$
p\left(z, e^{2 m} f(z)\right)=-\infty \quad \text { on } \mathbb{D}^{\mathrm{e}} .
$$

Since $e^{2 m} f(z)$ is holomorphic on $\mathbb{C} \backslash K$ and $\mathbb{C} \backslash K$ is connected, we get

$$
p\left(z, e^{2 m} f(z)\right)=-\infty \quad \text { on } \mathbb{D} .
$$

Hence, by Corollary 4 with $J$ in $\mathbb{D}$ and proceeding analogously as in the case $k=0$ we get

$$
p\left(z, e^{2 m+2} f(z)\right)=-\infty \quad \text { on } \mathbb{D}^{\mathrm{e}} \cup K_{0} .
$$

Therefore,

$$
p\left(z, F_{m+1}(z)\right)=-\infty \quad \text { on } \mathbb{D} \cup K_{0} \cup \mathbb{D}^{\mathrm{e}} .
$$

Now, set $k=-m-1$. We know that

$$
p\left(z, e^{-2 m-2} f(z)\right)=-\infty \quad \text { on } \mathbb{D}^{\mathrm{e}} .
$$

Hence, by the analyticity of $f$ on $\mathbb{C} \backslash K$ and the fact that $\mathbb{C} \backslash K$ is connected, we get

$$
p\left(z, e^{-2 m-2} f(z)\right)=-\infty \quad \text { on } \mathbb{D} .
$$

So, by Corollary 4 we get similarly

$$
p\left(z, e^{-2 m-4} f(z)\right)=-\infty \quad \text { on } \mathbb{D} \cup K_{0} \cup \mathbb{D}^{\mathrm{e}} .
$$


Consequently,

$$
p\left(z, F_{-m-1}(z)\right)=-\infty \quad \text { on } \mathbb{D} \cup K_{0} \cup \mathbb{D}^{\mathrm{e}} .
$$

Let us now consider the case $K=\partial \mathbb{D}$. We shall follow the same idea as in the previous case. First recall that $f_{j}$ is holomorphic on $\mathbb{C} \backslash K^{j}$. Let $p \in \operatorname{PSH}\left(\mathbb{C}^{2}\right)$ be such that $p\left(z, f_{1}(z)+f_{2}(z)\right)=-\infty, z \in \mathbb{D}^{\mathrm{e}}$. Then apply approximation of $f_{2}$ as in the previous case (with $K=K^{2}$ ). Then the sums of $f_{1}$ (holomorphic on $\mathbb{C} \backslash K^{1}$ ) with the elements of the approximating sequence of $f_{2}$ play the same role as the functions $f_{n}$ previously. Therefore, we get the equality $p\left(z, f_{1}(z)+e^{-2} f_{2}(z)\right)=-\infty, z \in \mathbb{D} \cup K_{0}^{2}$. Then applying the same reasoning for the function $f_{1}+e^{-2} f_{2}$ restricted this time to $\mathbb{D}$ (with $K=K^{1}$ and applying approximation of $\left.f_{1}\right)$ we get $p\left(z, e^{2} f_{1}(z)+e^{-2} f_{2}(z)\right)=-\infty$, $z \in \mathbb{D}^{\mathrm{e}}$, and $p\left(z, f_{1}(z)+e^{-2} f_{2}(z)\right)=-\infty, z \in K_{0}^{1}$. Proceeding inductively we get

$$
\begin{aligned}
p\left(z, e^{2 k} f_{1}(z)+e^{-2 k} f_{2}(z)\right) & =-\infty, \quad z \in \mathbb{D}^{\mathrm{e}}, k=0,1, \ldots, \\
p\left(z, e^{2 k} f_{1}(z)+e^{-2 k-2} f_{2}(z)\right) & =-\infty, \quad z \in K_{0}^{1} \cup K_{0}^{2} \cup \mathbb{D}, k=0,1, \ldots
\end{aligned}
$$

But proceeding exactly in the same way with $f_{1}$ replaced with $f_{2}$ (and vice versa) we get the desired equality for all $k \in \mathbb{Z}$.

REMARK 10. Let $f$ be as in the theorem for $K=\partial \mathbb{D}$. For $k \in \mathbb{Z}$ put

$$
F_{k}(z):= \begin{cases}e^{2 k} f_{1}(z)+e^{-2 k-2} f_{2}(z), & z \in \mathbb{D}, \\ e^{2 k} f_{1}^{*}(z)+e^{-2 k-2} f_{2}^{*}(z), & z \in K_{0}^{1} \cup K_{0}^{2}, \\ e^{2 k} f_{1}^{*}(z)+e^{-2 k} f_{2}(z), & z \in \mathbb{D}^{\mathrm{e}} .\end{cases}
$$

Then $\Gamma_{F_{k}}\left(\mathbb{D} \cup K \cup \mathbb{D}^{\mathrm{e}}\right) \subset\left(\Gamma_{f}(\mathbb{C} \backslash \partial \mathbb{D})\right)_{\mathbb{C}^{2}}^{*}$ and the functions $F_{k} \mid \mathbb{D}$ and $\left.F_{k}\right|_{\mathbb{D}^{\mathrm{e}}}$ are pseudocontinuations of each other across $K_{0}^{1} \cup K_{0}^{2}$, i.e. $\left(F_{k} \mid \mathbb{D}\right)^{*}(a)=$ $\left(F_{k} \mid \mathbb{D}^{\mathrm{e}}\right)^{*}(a), a \in K_{0}^{1} \cup K_{0}^{2}$.

6. Examples. It is natural to ask whether the assumption in Theorem 1 on the capacity of $K$ is essential, more precisely, whether there are compact sets $K \subset \partial \mathbb{D}$ such that $m(K)>0$ and $\operatorname{cap}(\partial \mathbb{D} \backslash K)=1$. Below we shall produce such a set. First we prove

LEMMA 11. There exists an $F_{\sigma}$-set $F \subset \partial \mathbb{D}$ such that $m(F)=0$ and $\operatorname{cap}(F)=1$.

Proof. First recall that for any Borel subset $I \subset \partial \mathbb{D}$ and all $n \in \mathbb{N}$, if we set $\widetilde{I}:=\left\{\lambda \in \partial \mathbb{D}: \lambda^{n} \in I\right\}$, then

$$
m(\widetilde{I})=m(I), \quad \operatorname{cap}(\widetilde{I})=\sqrt[n]{\operatorname{cap} I}
$$

There exists a compact set $K \subset \partial \mathbb{D}$ such that $m(K)=0$ and $\operatorname{cap}(K)>0$ (take for example a Cantor type set on the unit circle). Put $I_{k}=\{z \in \partial \mathbb{D}$ : $\left.z^{k} \in K\right\}$. It is sufficient to define $F=\bigcup_{k=1}^{\infty} I_{k}$. 
EXAMPLE 12. Let $0<\delta<1$. Then there is a compact set $K \subset \partial \mathbb{D}$ such that $m(\partial \mathbb{D} \backslash K)<\delta$ (equivalently, $m(K)>1-\delta$ ) and $\operatorname{cap}(\partial \mathbb{D} \backslash K)=1$.

Proof. Take an $F_{\sigma}$-set $F \subset \partial \mathbb{D}$ such that $m(F)=0$ and $\operatorname{cap}(F)=1$. For any $\delta>0$ there exists an open set $U_{\delta} \subset \partial \mathbb{D}$ such that $F \subset U_{\delta}$ and $m\left(U_{\delta}\right)<\delta$. Put $K_{\delta}=\partial \mathbb{D} \backslash U_{\delta}$.

It seems to us that the most interesting examples of compact sets satisfying the conditions of Theorem 1 are ones which are totally disconnected. Below we show the existence of such subsets of $\partial \mathbb{D}$.

EXAMPLE 13. There is a totally disconnected compact set $K \subset \partial \mathbb{D}$ as desired in Theorem 1, i.e. such that $\operatorname{cap}(\partial \mathbb{D} \backslash K)<1$ and $m(K \cap U)>0$ for any $z \in K$ and any neighborhood $U$ of $z$.

The construction of $K$ is inductive and standard. For example we may define $K:=\partial \mathbb{D} \backslash \bigcup_{n=1}^{\infty} L_{n}$, where $L_{n}$ is a union of disjoint open arcs with centers at $e^{i j \pi / 2^{n}}$, where $j=-\left(2^{n}-1\right),-\left(2^{n}-3\right), \ldots, 2^{n}-1$ with $m$-measure of each arc smaller than $1 / 2^{2 n}$ and with $\operatorname{cap}\left(L_{1} \cup \cdots \cup L_{n}\right)<1 / 2$.

Then $\operatorname{cap}(\partial \mathbb{D} \backslash K) \leq 1 / 2$ and the choice may be done so that $m(U \cap K)>0$ for any $z \in K$ and for any neighborhood $U$ of $z$.

In fact, we may even specify effectively how large the open arcs deleted in the above process may be. These lengths may be deduced from the following example. that

EXAMPLE 14. Let $\left\{I_{j}\right\}_{j=1}^{\infty}$ be a sequence of disjoint open arcs in $\partial \mathbb{D}$ such

$$
\sum_{j=1}^{\infty} \frac{1}{\log \left(2 / m\left(I_{j}\right)\right)}<\infty
$$

(for instance $m\left(I_{j}\right)=\exp \left(-j^{2}\right)$ ). Then there is a $j_{0}$ such that $U:=\bigcup_{j=j_{0}}^{\infty} I_{j}$ has the properties

$$
\operatorname{cap}(U)<1, \quad m(\partial \mathbb{D} \backslash U)>0 .
$$

In fact, the result follows immediately from the subadditivity property of the logarithmic capacity and the fact that $\operatorname{cap}\left(I_{j}\right)=\sin \left(m\left(I_{j}\right) \pi / 4\right)$.

\section{References}

[1] E. Chirka, Complex Analytic Sets, Kluwer, 1989.

[2] A. Edigarian and J. Wiegerinck, The pluripolar hull of graphs of certain holomorphic functions, Indiana Univ. Math. J. 52 (2003), 1663-1680.

[3] -, - Determination of the pluripolar hull of graphs of certain holomorphic functions, Ann. Inst. Fourier (Grenoble) 54 (2004), 2085-2104.

[4] T. Edlund and B. Jöricke, The pluripolar hull of a graph and fine analytic continuation, Ark. Mat., to appear. 
[5] B. Josefson, On the equivalence between locally polar and globally polar sets for plurisubharmonic functions on $\mathbb{C}^{n}$, ibid. 16 (1978), 109-115.

[6] M. Klimek, Pluripotential Theory, London Math. Soc. Monogr. 6, Clarendon Press, 1991.

[7] N. Levenberg and E. Poletsky, Pluripolar hulls, Michigan Math. J. 46 (1999), 151-162.

[8] E. Poletsky and J. Wiegerinck, Graphs of multiple sheeted pluripolar hulls, Ann. Polon. Math. 88 (2006), 161-171.

[9] T. Ransford, Potential Theory in the Complex Plane, London Math. Soc. Student Texts 28, Cambridge Univ. Press, 1995.

[10] J. Siciak, Pluripolar continuation of graphs of Blaschke products, in: Proc. 12th Internat. Conf. on Finite or Infinite Dimensional Complex Analysis and Applications, Kyushu Univ. Press, 2005, 305-317.

[11] M. Tsuji, Potential Theory in Modern Function Theory, Maruzen, Tokyo, 1959.

[12] L. Zalcman, Analytic Capacity and Rational Approximation, Springer, 1968.

[13] A. Zeriahi, Ensembles pluripolaires exceptionnels pour la croissance partielle des fonctions holomorphes, Ann. Polon. Math. 50 (1989), 11-91.

[14] W. Zwonek, A note on pluripolar hulls of graphs of Blaschke products, Potential Anal. 22 (2005), 195-206.

Institute of Mathematics

Jagiellonian University

Reymonta 4

30-059 Kraków, Poland

E-mail: Armen.Edigarian@im.uj.edu.pl

Jozef.Siciak@im.uj.edu.pl

Wlodzimierz.Zwonek@im.uj.edu.pl

Received May 16, 2005

Revised version June 20, 2006 